\title{
Toll-like receptor 2 promotes bacterial clearance during the initial stage of pulmonary infection with Acinetobacter baumannii
}

\author{
CHANG-HWAN KIM ${ }^{1 *}$, DONG-JAE KIM ${ }^{2 *}$, SANG-JIN LEE ${ }^{2}$, YU-JIN JEONG ${ }^{2}$, MIN-JUNG KANG ${ }^{2}$, \\ JUN-YOUNG LEE ${ }^{2}$, JIN-A CHOI ${ }^{2}$, SUN-JUNG KWON ${ }^{3}$, JAE-HAK PARK ${ }^{1}$ and JONG-HWAN PARK ${ }^{2}$ \\ ${ }^{1}$ Department of Laboratory Animal Medicine, College of Veterinary Medicine, Seoul National University, \\ Seoul 151-742; Departments of ${ }^{2}$ Biochemistry and ${ }^{3}$ Internal Medicine, College of Medicine, \\ Konyang University, Daejeon 302-718, Republic of Korea
}

Received July 15, 2013; Accepted February 5, 2014

DOI: $10.3892 / \mathrm{mmr} .2014 .1966$

\begin{abstract}
Toll-like receptor 2 (TLR2) has been identified as a sensor for bacterial lipoprotein. To determine the role of TLR2 in host defense against Acinetobacter baumannii infection, wild-type (WT) and TLR2-deficient mice were infected intranasally with A. baumannii. Body weight, cytokine and chemokine levels in bronchoalveolar (BAL) fluid and lung histopathology were examined. Body weight changes in TLR2-deficient mice were comparable to those of WT mice throughout the experimental period. However, TLR2-deficient mice exhibited an increased bacterial load in the lungs and increased levels of interleukin- 6 and chemokine (C-X-C motif) ligand 2 in BAL fluids compared with WT mice 1 day after infection. Histopathological features of lung tissue in WT and TLR2-deficient mice were comparable between WT and TLR2-deficient mice. Results of the present study demonstrate that TLR2 may have a minimal role in the host defense against A. baumannii at the early stages of infection.
\end{abstract}

\section{Introduction}

Acinetobacter baumannii has emerged as a major causative agent of nosocomial infection in hospitals worldwide $(1,2)$. According to the data from a national surveillance study performed in the United States, A. baumannii was shown to be the etiological agent in $6.9 \%$ of nosocomial pneumonias in 2004, which was an $\sim 72 \%$ increase compared with data collected in 1986 (3). Pulmonary compartments are the most favored site of A. baumannii infection, and

Correspondence to: Professor Jong-Hwan Park, Department of Biochemistry, College of Medicine, Konyang University, 158 Gwanjeodong-ro, Seo-gu, Daejeon 302-718, Republic of Korea E-mail: jonpark@konyang.ac.kr

*Contributed equally

Key words: toll-like receptor 2, Acinetobacter baumannii, bacterial clearance, lung ventilatory-associated and community-acquired pneumonia are extremely common. The mortality of ventilatory-associated pneumonias due to A. baumannii may be as high as $75 \%$ (4). Furthermore, emergence of multi-drug resistant $A$. baumannii has become a significant health concern leading to limited therapeutic options in recent decades $(1,2)$. The increased incidence of antibiotic resistance in these bacteria underlines the importance of understanding the host immune response against $A$. baumannii to prevent and control infection.

Innate immune systems provide the first line of defense against pathogenic bacteria, by recognition of pathogen-associated molecular patterns. Toll-like receptors (TLRs) are the best-studied family of pattern recognition receptors (PRRs) and are comprised of $\mathrm{N}$-terminal leucine-rich repeats, a transmembrane domain and a C-terminal Toll/interleukin (IL)-1 receptor domain. TLRs $1,2,4,5$ and 6 are present on the cell surface, whereas TLRs 3, 7 and 9 are localized in the endosome (5). Following the recognition of specific molecular patterns found in microbial pathogens, TLRs trigger downstream signaling pathways [myeloid differentiation primary-response protein 88 (MyD88)-dependent and/or TIR domain-containing adapter-inducing interferon- $\beta$-dependent pathways], and produce proinflammatory cytokines and antimicrobial factors to eradicate the invading pathogens. Engagement of TLRs initiates signaling through intracellular pathways that lead to activation of nuclear factor- $\kappa \mathrm{B}(\mathrm{NF}-\kappa \mathrm{B})$, mitogen-activated protein kinases (MAPKs) and interferon regulatory factors $(6,7)$.

TLR4 is critical for host defense against Gram-negative bacterial infection as it recognizes lipopolysaccharide (LPS) (7). TLR4 mediates bacterial clearance and production of proinflammatory cytokines and chemokines in A. baumannii infection (8). In addition to TLR4, TLR2 is involved in host innate immunity against microbial infections by recognizing peptidoglycans of the bacterial cell wall and activating $\mathrm{NF}-\kappa \mathrm{B}$ and MAPKs through the MyD88-dependent pathway (9-11). Although A. baumannii is known to induce TLR2-stimulatory activity $(12,13)$, the role of TLR2 in A. baumannii-induced immune responses remains unclear. Previously, an in vivo study demonstrated that TLR2 deficiency leads to improved bacterial clearance and increased production of chemokines and myeloperoxidase in the lungs of mice infected with A. baumannii (8). By contrast, TLR2 is essential for optimal immune responses 


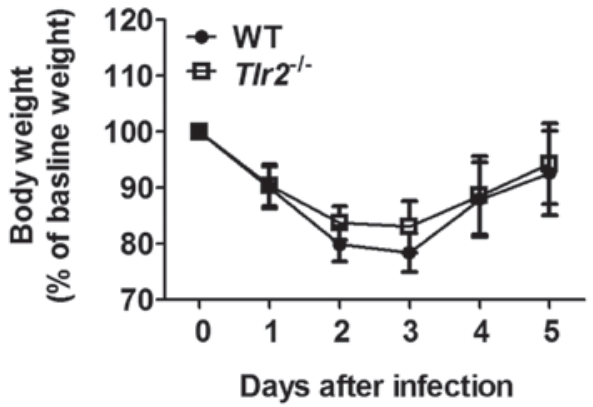

Figure 1. Body weight changes with A. baumannii infection in WT and TLR2-deficient mice. WT and TLR2-deficient mice were infected intranasally with $A$. baumannii $\left(3 \times 10^{7} \mathrm{CFU}\right)$ and body weight changes were monitored daily. WT, wild-type; TLR2, toll-like receptor 2; CFU, colony-forming units.

against $A$. baumannii in macrophages and lung epithelial cells $(12,14)$. Therefore, the present study sought to confirm the role of TLR2 in bacterial colonization, cytokine and chemokine production and lung histopathology, by A. baumannii infection in mice.

\section{Materials and methods}

Mice. TLR2-deficient mice on C57BL/6 background were purchased from Jackson Laboratory (Bar Harbor, ME, USA) and wild-type (WT) C57BL/6 mice were from Koatech (Pyeongtaek, Korea). The animal studies were conducted under approved protocols by the Institutional Animal Care and Use Committee of Konyang University (Daejeon, Korea).

Bacterial infection. A. baumannii strain KCCM 35453 (ATCC 15150) was purchased from Korean Culture Center of Microorganisms (Seoul, Korea). For bacterial preparation, single colonies were inoculated into $5 \mathrm{ml}$ Luria Bertani (LB) broth and grown overnight at $37^{\circ} \mathrm{C}$ under agitation. A 1:5 dilution of the culture grown overnight was allowed to grow in fresh medium at $37^{\circ} \mathrm{C}$ under agitation for a further $2 \mathrm{~h}$. Bacteria were washed and resuspended with sterile phosphate-buffered saline (PBS) to $10^{9}$ colony-forming units (CFU)/ml. Mice were anesthetized by intraperitoneal injection of $10 \mathrm{mg} / \mathrm{kg}$ rompun (Bayer Korea Ltd., Seoul, Korea) and 50 mg/kg zoletil (Virbac Korea Co., Ltd., Seoul, Korea), and $30 \mu \mathrm{l}$ prepared bacteria was inoculated intranasally. Infected mice were monitored daily for body weight changes and clinical signs. At 1, 3 and 5 days after inoculation, mice were sacrificed and bronchoalveolar lavage (BAL) fluid was collected.

Bacterial count in lung tissue. Following BAL fluid collection, the right lobes of lungs were collected in sterile PBS for bacterial counting. The extracted lobes were weighed, homogenized, serially diluted and $100 \mu$ l homogenate was spread onto LB agar plates. Following overnight culture in a $37^{\circ} \mathrm{C}$ incubator, the colony was counted and the number of bacteria (measured as $\mathrm{CFU} / \mathrm{g}$ lung tissue) was calculated.

Measurement of cytokines and chemokines. The levels of IL-6, tumor necrosis factor- $\alpha$ (TNF- $\alpha$ ), chemokine (C-X-C motif) ligand 2 (CXCL2) and chemokine (C-C motif) ligand 2
(CCL2) from BAL fluid of A. baumannii-infected mice were determined using commercial ELISA kits (R\&D Systems, Minneapolis, MN, USA).

Histopathological examination. The left lobes of lungs were harvested and fixed in $10 \%$ neutral formalin for histopathological observation. The tissues were routinely processed with alcohol and xylene series and embedded in paraffin. Subsequently, 3- $\mu \mathrm{m}$ sections were prepared, stained with hematoxylin and eosin and examined by microscopy.

Statistical analysis. The differences in mean values among different groups were tested and the values were expressed as the mean \pm standard deviation. All of the statistical calculations were carried out using Microsoft Excel (Microsoft, Redmond, WA, USA). $\mathrm{P}<0.05$ was considered to indicate a statistically significant difference.

\section{Results}

Clinical signs and body weight changes by A. baumannii infection in mice. A. baumannii infection caused mild physical signs of ill health in WT and TLR2-deficient mice, manifested as weight loss, ruffled fur, decreased movement and huddling. These were without any significant difference 1 day after infection (data not shown). These clinical signs improved from 3 days after infection. The weight loss was prominent in the early stages of infection and continued until 3 days after infection. Following this, the majority of mice gradually regained body weight and had recovered to within a normal range by 5 days after infection (Fig. 1).

Bacterial clearance in the lungs of mice infected with A. baumannii. To determine the role of TLR2 in bacterial clearance in vivo, the lung tissue was extracted from A. baumannii-infected WT and TLR2-deficient mice and the number of bacteria was counted. The pulmonary bacterial loads were significantly higher in TLR2-deficient mice compared with WT mice 1 day after infection (Fig. 2A). However, at 3 and 5 days after infection, the loads were comparable in WT and TLR2-deficient mice (Fig. 2B and C). These results indicate that TLR2 may contribute to bacterial clearance in A. baumannii-infected mice at the early stages of infection but not at the later stages.

Production of cytokines and chemokines by A. baumannii in BAL fluids. To examine whether TLR2 has a role in the production of cytokines and chemokines following A. baumannii infection, levels of cytokines (IL-6 and TNF- $\alpha$ ) and chemokines (CXCL2 and CCL2) from BAL fluids of A. baumannii-infected mice were measured 1, 3 and 5 days after infection. The levels of IL- 6 and CXCL2 were significantly higher in the BAL fluid of TLR2-deficient mice than in WT mice 1 day after infection (Fig. 3A and B). Although there was no statistically significant difference, the TNF- $\alpha$ level was also slightly higher in the BAL fluid of TLR2-deficient mice compared with WT mice (Fig. 3C).

Histopathology of the lung of A. baumannii-infected mice. Histopathological features of lung tissue from WT and 

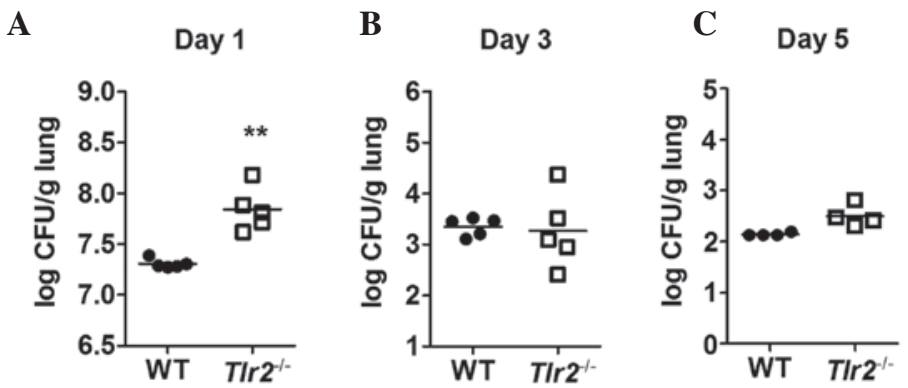

Figure 2. Bacterial clearance in the lung of mice infected with A. baumannii. Bacterial numbers of WT and TLR2-deficient mice were measured in lung homogenates (A) 1, (B) 3 and (C) 5 days after infection. ${ }^{*}<0.01$, vs. WT group. WT, wild-type; TLR2, toll-like receptor 2.
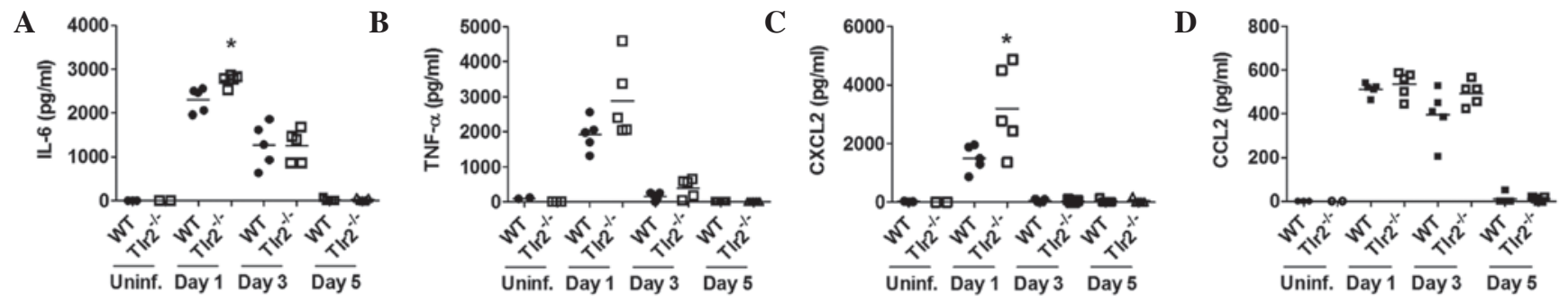

Figure 3. The production of cytokines and chemokines by A. baumannii in BAL fluids. WT and TLR2-deficient mice were infected with A. baumannii and BAL fluids were collected 1, 3 and 5 days after infection. The levels of (A) IL-6, (B) TNF- $\alpha$, (C) CXCL2 and (D) CCL2 in BAL fluids were measured by ELISA. "P<0.01, vs. WT group. BAL, bronchoalveolar lavage; TLR-2, toll-like receptor 2; IL-6, interleukin-6; TNF- $\alpha$, tumor necrosis factor- $\alpha$; CXCL2, chemokine (C-X-C motif) ligand 2; CCL2, chemokine (C-C motif) ligand 2; WT, wild-type.

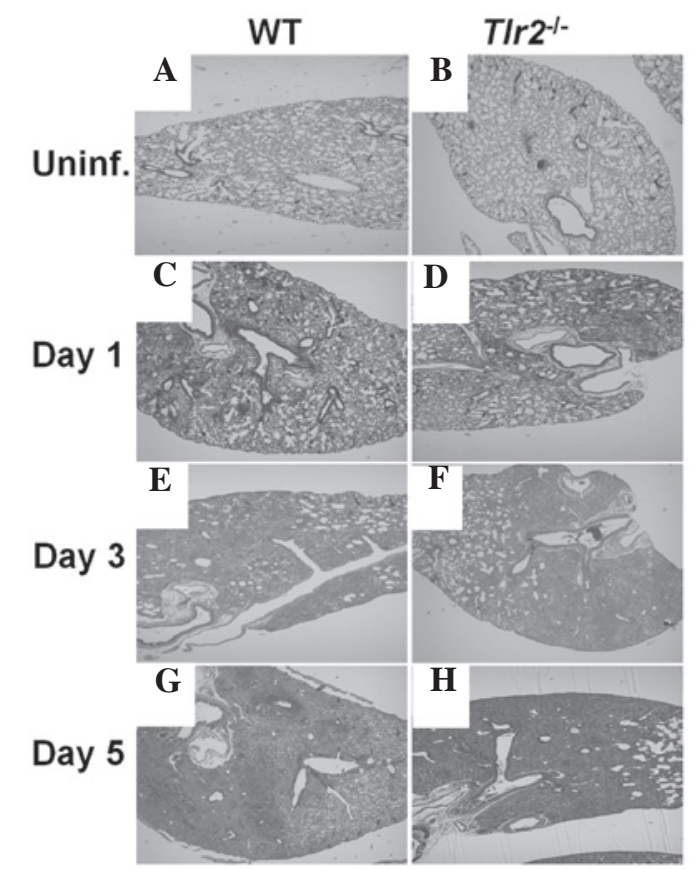

Figure 4. Histopathology in the lung of A. baumannii-infected mice. WT and TLR2-deficient mice were infected with $3 \times 10^{7} \mathrm{CFU}$ A. baumannii and representative histological images of the lungs are depicted (hematoxylin and eosin; magnification, x40). WT, wild-type; TLR2, toll-like receptor 2; CFU, colony-forming units.

TLR2-deficient mice was assessed 1, 3 and 5 days after inoculation with A. baumannii. Whilst no infiltration of inflammatory cells was observed prior to A. baumannii infection in the lungs of WT and TLR2-deficient mice (Fig. 4A and B), infection led to polymorphonuclear (PMN) cell infiltration in the alveolar spaces and perivascular and peribronchiolar areas in WT and TLR2-deficient mice from 1 day after infection (Fig. 4C and D). Infiltration of these cells became severe at 3 (Fig. 4E and F) and 5 days (Fig. 4G and H) after infection, and no marked difference in inflammatory cell infiltration was observed between WT and TLR2-deficient mouse lung tissue (Fig. 4). These results indicate that TLR2 may not be involved in the progression of lung inflammation in mice.

\section{Discussion}

Inflammatory responses play a pivotal role in lung defense against bacterial pathogens, including A. baumannii. This specific response includes the release of pro-inflammatory cytokines and chemokines $(8,15,16)$. IL- 6 , TNF- $\alpha$, and IL- $1 \beta$ are representative proinflammatory cytokines and are associated with the upregulation of cell adhesion molecules and antigen presentation. IL-8 (keratinocyte-derived protein chemokine/CXCL1 in mice), monocyte chemotactic protein-1/CCL2, and macrophage inflammatory protein-2/CXCL2 act as chemoattractants and are required for recruitment of immune cells from the bloodstream into the airway (17). In addition to immune cells, airway epithelial cells contribute to host resistance against $A$. baumannii by producing antimicrobial peptides, for example defensins $(18,19)$. All of these factors are closely related and cooperate during the immune response against $A$. baumannii.

In the present study, mice lacking functional TLR2 exhibited a selective impaired bacterial clearance compared with 
WT mice 1 day after infection, although lung infiltration of immune cells was comparable between the two groups. These results are in accordance with a previous report demonstrating that TLR2 deficiency impaired the nitric oxide-mediated bacterial killing capacity of PMN cells and alveolar macrophages, without any defects in the immune cell influx in pulmonary infection of Porphyromonas gingivalis (20). In addition, the possible role of TLR2-dependent local immune responses cannot be excluded. Our previous study revealed that TLR2 is not required for cytokine production in macrophages in response to A. baumannii (21). However, A. baumannii may induce IL- 8 production in human lung epithelial cells and was inhibited by TLR2 small interfering RNA (12). Although TLR2 deficiency demonstrated increased CXCL2 production 1 day after infection, no significant difference was observed between the two groups with regard to PMN cell infiltration into the lung. It is possible that TLR2-independent mechanisms may have compensated for this function, for example stimulation by bacterial-derived formyl peptides of the formyl-peptide receptor, which plays a major role in PMN recruitment to infected alveoli (22).

A number of studies have reported that TLR2-deficient mice exhibit increased resistance to pulmonary infection with A. baumannii (8) or pilA mutant Pseudomonas aeruginosa (23), and to systemic challenge with Yersinia enterocolitica (24) or Candida albicans (25). It is hypothesized that these results may be due to TLR2 signaling having several inhibitory effects on the inflammatory response, by inducing IL-10 or downregulating TLR4 expression. However, the distinct mechanisms have not been clearly identified. In the present study, no bacterial clearance defects were observed in the lungs of TLR2-deficient mice compared with those of WT mice 3 and 5 days after infection. In addition, TLR2-deficient mice exhibited similar inflammatory cell infiltration in the lungs as WT mice with A. baumannii, which was not consistent with the previous report (8). This discrepancy may be due to the distinct bacterial origin and different experimental conditions.

Although TLR4 is regarded as the main PRR of Gram-negative bacteria, TLR2 has also been reported to participate in the recognition of some Gram-negative bacteria, including Helicobacter pylori, Porphyromonas gingivalis and Bacteroides fragilis $(11,26,27)$. Therefore, TLR2 serves as a functional receptor for Gram-positive and -negative bacteria and induces production of proinflammatory cytokines (28). In conclusion, results of the present study demonstrated that TLR2 may be involved in the recognition of A. baumannii, particularly at the early stages of infection. A previous study revealed that LPS-deficient $A$. baumannii exhibited impaired ability to produce TNF- $\alpha$ in TLR2-deficient macrophages compared with WT cells (14). Therefore, it is necessary to re-analyze the in vivo effects of TLR2, using an LPS-deficient strain of $A$. baumannii or by comparing phenotypes between TLR4- and TLR2/4-deficient mice.

\section{Acknowledgements}

This study was supported by a program for general research from the National Research Foundation of Korea (no. NRF-2012R1A1A2041944).

\section{References}

1. Perez F, Hujer AM, Hujer KM, Decker BK, Rather PN and Bonomo RA: Global challenge of multidrug-resistant Acinetobacter baumannii. Antimicrob Agents Chemother 51: 3471-3484, 2007.

2. Van Looveren M and Goossens H; ARPAC Steering Group: Antimicrobial resistance of Acinetobacter spp. in Europe. Clin Microbiol Infect 10: 684-704, 2004.

3. Gaynes R and Edwards JR; National Nosocomial Infectious Surveillance System: Overview of nosocomial infections caused by gram-negative bacilli. Clin Infect Dis 41: 848-854, 2005.

4. Fagon JY, Chastre J, Domart Y, Trouillet JL and Gibert C: Mortality due to ventilator-associated pneumonia or colonization with Pseudomonas or Acinetobacter species: assessment by quantitative culture of samples obtained by a protected specimen brush. Clin Infect Dis 23: 538-542, 1996.

5. Akira S: Innate immunity to pathogens: diversity in receptors for microbial recognition. Immunol Rev 227: 5-8, 2009.

6. Kawai T and Akira S: TLR signaling. Cell Death Differ 13: 816-825, 2006.

7. Takeda $\mathrm{K}$ and Akira S: Toll-like receptors in innate immunity. Int Immunol 17: 1-14, 2005.

8. Knapp S, Wieland CW, Florquin S, et al: Differential roles of CD14 and toll-like receptors 4 and 2 in murine Acinetobacter pneumonia. Am J Respir Crit Care Med 173: 122-129, 2006.

9. Flo TH, Halaas O, Lien E, et al: Human toll-like receptor 2 mediates monocyte activation by Listeria monocytogenes, but not by group B streptococci or lipopolysaccharide. J Immunol 164: 2064-2069, 2000.

10. Echchannaoui H, Frei K, Schnell C, Leib SL, Zimmerli W and Landmann R: Toll-like receptor 2-deficient mice are highly susceptible to Streptococcus pneumoniae meningitis because of reduced bacterial clearing and enhanced inflammation. J Infect Dis 186: 798-806, 2002.

11. Smith MF Jr, Mitchell A, Li G, et al: Toll-like receptor (TLR) 2 and TLR5, but not TLR4, are required for Helicobacter pylori-induced $\mathrm{NF}-\kappa \mathrm{B}$ activation and chemokine expression by epithelial cells. J Biol Chem 278: 32552-32560, 2003

12. March C, Regueiro V, Llobet E, et al: Dissection of host cell signal transduction during Acinetobacter baumannii-triggered inflammatory response. PloS One 5: e10033, 2010.

13. Erridge C, Moncayo-Nieto OL, Morgan R, Young M and Poxton IR: Acinetobacter baumannii lipopolysaccharides are potent stimulators of human monocyte activation via Toll-like receptor 4 signalling. J Med Microbiol 56: 165-171, 2007.

14. Moffatt JH, Harper M, Mansell A, et al: Lipopolysaccharide-deficient Acinetobacter baumannii shows altered signaling through host Toll-like receptors and increased susceptibility to the host antimicrobial peptide LL-37. Infect Immun 81: 684-689, 2013.

15. Qiu H, KuoLee R, Harris G and Chen W: High susceptibility to respiratory Acinetobacter baumannii infection in A/J mice is associated with a delay in early pulmonary recruitment of neutrophils. Microbes Infect 11: 946-955, 2009.

16. Van Faassen H, KuoLee R, Harris G, Zhao X, Conlan JW and Chen W: Neutrophils play an important role in host resistance to respiratory infection with Acinetobacter baumannii in mice. Infect Immun 75: 5597-5608, 2007.

17. Craig A, Mai J, Cai S and Jeyaseelan S: Neutrophil recruitment to the lungs during bacterial pneumonia. Infect Immun 77: 568-575, 2009.

18. Routsias JG, Karagounis P, Parvulesku G, Legakis NJ and Tsakris A: In vitro bactericidal activity of human beta-defensin 2 against nosocomial strains. Peptides 31: 1654-1660, 2010.

19. Maisetta G, Batoni G, Esin S, et al: In vitro bactericidal activity of human $\beta$-defensin 3 against multidrug-resistant nosocomial strains. Antimicrob Agents Chemother 50: 806-809, 2006.

20. Hajishengallis G, Wang M, Bagby GJ and Nelson S: Importance of TLR2 in early innate immune response to acute pulmonary infection with Porphyromonas gingivalis in mice. J Immunol 181: 4141-4149, 2008.

21. Kim CH, Jeong YJ, Lee J, et al: Essential role of toll-like receptor 4 in Acinetobacter baumannii-induced immune responses in immune cells. Microb Pathog 54: 20-25, 2013.

22. Fillion I, Ouellet N, Simard M, Bergeron Y, Sato S and Bergeron MG: Role of chemokines and formyl peptides in pneumococcal pneumonia-induced monocyte/macrophage recruitment. J Immunol 166: 7353-7361, 2001. 
23. Lorenz E, Chemotti DC, Vandal K and Tessier PA: Toll-like receptor 2 represses nonpilus adhesin-induced signaling in acute infections with the Pseudomonas aeruginosa pilA mutant. Infect Immun 72: 4561-4569, 2004.

24. Sing A, Rost D, Tvardovskaia N, et al: Yersinia V-antigen exploits Toll-like Receptor 2 and CD14 for interleukin 10-mediated immunosuppression. J Exp Med 196: 1017-1024, 2002.

25. Netea MG, Sutmuller R, Hermann C, et al: Toll-like receptor 2 suppresses immunity against Candida albicans through induction of IL-10 and regulatory T cells. J Immunol 172: 3712-3718, 2004.
26. Darveau RP,Pham TT,Lemley K, et al: Porphyromonas gingivalis lipopolysaccharide contains multiple lipid A species that functionally interact with both toll-like receptors 2 and 4 . Infect Immun 72: 5041-5051, 2004.

27. Erridge C, Pridmore A, Eley A, Stewart J and Poxton IR: Lipopolysaccharides of Bacteroides fragilis, Chlamydia trachomatis and Pseudomonas aeruginosa signal via toll-like receptor 2. J Med Microbiol 53: 735-740, 2004

28. Dziarski R and Gupta D: Role of MD-2 in TLR2- and TLR4-mediated recognition of Gram-negative and Gram-positive bacteria and activation of chemokine genes. J Endotoxin Res 6: 401-405, 2000. 